Supporting Information for "Precise, self-limited epitaxy of ultrathin organic semiconductors and heterojunctions tailored by van der Waals interactions"

Bing Wu, ${ }^{1, \dagger}$ Yinghe Zhao, ${ }^{2, \dagger}$ Haiyan Nan, ${ }^{2, \dagger}$ Ziyi Yang, ${ }^{1}$ Yuhan Zhang, ${ }^{1}$ Huijuan Zhao, ${ }^{1}$ Daowei He, ${ }^{1}$ Zonglin Jiang, ${ }^{1}$ Xiaolong Liu, ${ }^{1}$ Yun Li, ${ }^{1}$ Yi Shi, ${ }^{1, *}$ Zhenhua Ni, Jinlan Wang, ${ }^{2,3} *$ Jian-Bin $\mathrm{Xu}^{4}$ \& Xinran Wang ${ }^{1, *}$

${ }^{1}$ National Laboratory of Solid State Microstructures, School of Electronic Science and Engineering, and Collaborative Innovation Center of Advanced Microstructures, Nanjing University, Nanjing 210093, China

${ }^{2}$ Department of Physics, Southeast University, Nanjing 211189, P. R. China

3Synergetic Innovation Center for Quantum Effects and Applications (SICQEA), Hunan Normal University, Changsha 410081, China

${ }^{4}$ Department of Electronic Engineering and Materials Science and Technology Research Center, The Chinese University of Hong Kong, Hong Kong SAR, P. R. China

* Correspondence should be addressed to X. W. (xrwang@nju.edu.cn), Y. S. (yshi@,nju.edu.cn) or J. W. (jlwang@seu.edu.cn)

$\dagger$ These authors contribute equally to this work. 


\section{SLOMBE of $\mathrm{C}_{8}$-BTBT, PTCDA and heterojunctions}

We used mechanically exfoliated graphene and $\mathrm{BN}$ on $300 \mathrm{~nm} \mathrm{SiO}_{2} / \mathrm{Si}$ as the epitaxy substrate. Before growth, the graphene and $\mathrm{BN}$ sheet was characterized by optical microscope and AFM to obtain the thickness and topological information. The growth of $\mathrm{C}_{8}-\mathrm{BTBT}$ and PTCDA was carried out in a home-built single-zone vacuum tube furnace. We placed the $\mathrm{C}_{8^{-}}$ BTBT source powder in the centre of the heating zone and the graphene and BN downstream. We used a turbo molecular pump to evacuate the quartz tube to $\sim 4 \times 10^{-6}$ Torr. We then heated up the source powders to a target temperature $\left(110 \sim 120^{\circ} \mathrm{C}\right.$ for $\mathrm{C}_{8}$-BTBT, $270 \sim 280^{\circ} \mathrm{C}$ for PTCDA) to start the growth. To achieve SL epitaxy of monolayer PTCDA as shown in Fig. 4, the graphene substrate was placed $2 \sim 4 \mathrm{~cm}$ away from the centre.

\section{AFM, KPFM, Raman spectroscopy, polarization-dependent absorption and PL measurements}

AFM (both regular and high-resolution) and KPFM were performed on an Asylum Cypher under ambient conditions. We used Asylum ARROW UHF tips for high-resolution AFM.

Raman spectroscopy was performed on a LabRAM HR800 Raman system with 514nm laser excitation. The Raman mapping in Fig. 2 was performed on a WITec Alpha 300R confocal Raman system with a 532nm laser excitation (spot size $\sim 300 \mathrm{~nm}$, laser power $1 \mathrm{~mW}$ ).

PL measurement was performed on a LabRAM HR800 Raman system with 514nm laser excitation (the laser power at sample is kept below $10 \mu \mathrm{W}$ ).

Polarization-dependent absorption measurement was performed on a WITec Alpha 300R system with two linear polarizers (one between illumination source and sample, and the other between sample and detector), and without the notch filter. The two polarizers were 
approximately cross-polarized to minimize the background signal of $\mathrm{SiO}_{2}$. White light was illuminated on the sample through a $50 \times$ objective lens, and the reflected light was collected by a CCD camera through a spectrometer. The images were obtained by scanning the sample with step size of $500 \mathrm{~nm}$. We plotted the images by integrating the spectrum from 520 to $550 \mathrm{~nm}$.

\section{Details of MD calculations}

The MD simulations are performed in GROMACS-5.0.1 package $^{1}$ under the ensemble of constant particle number, volume, and temperature. The time step of 1 fs and cut-off distance of $1.5 \mathrm{~nm}$ are used. Outside the range, the smoothed particle mesh Ewald sum is applied to deal with the long-range coulomb interaction ${ }^{2}$. The temperature is controlled by using the berendsen thermostat and periodic boundary conditions are employed to avoid the edge effects.

The force field parameters are obtained by using AnteChamber PYthon Parser interface ${ }^{3}$ on the basis of all-atom AMBER99SB force field ${ }^{4}$, which can be found in supporting information. The interlayer interaction is described by combining Lennard-Jones 12-6 and Coulomb potentials:

$$
E=\sum_{i=1}^{M} \sum_{j=1}^{N}\left[\frac{\left(\mathrm{C}_{i}^{(12)} C_{j}^{(12)}\right)^{1 / 2}}{r_{i j}^{12}}-\frac{\left(\mathrm{C}_{i}^{(6)} C_{j}^{(6)}\right)^{1 / 2}}{r_{i j}{ }^{6}}\right]+k_{e} \frac{q_{i} q_{j}}{r_{i j}}
$$

where $\mathrm{C}^{(12)}, \mathrm{C}^{(6)}$ and $\mathrm{q}$ represent atomic vdW parameters and partial charge, respectively; $\mathrm{r}$ is the interatomic distance and $\mathrm{k}_{\mathrm{e}}$ is the electrostatic constant.

\section{Device fabrication and measurements}

To fabricate the device in Fig. 5, we started by exfoliating graphene on $300 \mathrm{~nm} \mathrm{SiO}_{2} / \mathrm{Si}$ substrate. We then transferred a 100nm thick Au electrode to cover part of the graphene as 
bottom electrode. PTCDA $(\sim 6 \mathrm{~nm})$ and $\mathrm{C}_{8}$-BTBT $(\sim 9 \mathrm{~nm})$ were grown on the graphene sequentially. Finally we transferred another $\mathrm{Au}$ film on $\mathrm{C}_{8}$ - $\mathrm{BTB}$ as the top electrode. The transfer of Au electrode was performed under an optical microscope using a tungsten probe tip attached to a micro manipulator ${ }^{7}$.

Electrical measurements were carried out by an Agilent B1500 semiconductor parameter analyser in a probe station under ambient condition. The photoresponse characteristics were investigated under $514 \mathrm{~nm}$ laser excitation. The laser (spot size $\sim 1 \mu \mathrm{m}$ ) was focused on the sample with a $50 \times$ objective $(\mathrm{NA}=0.5)$.

\section{Additional data of $\mathrm{C}_{8}-\mathrm{BTBT}$ and PTCDA}
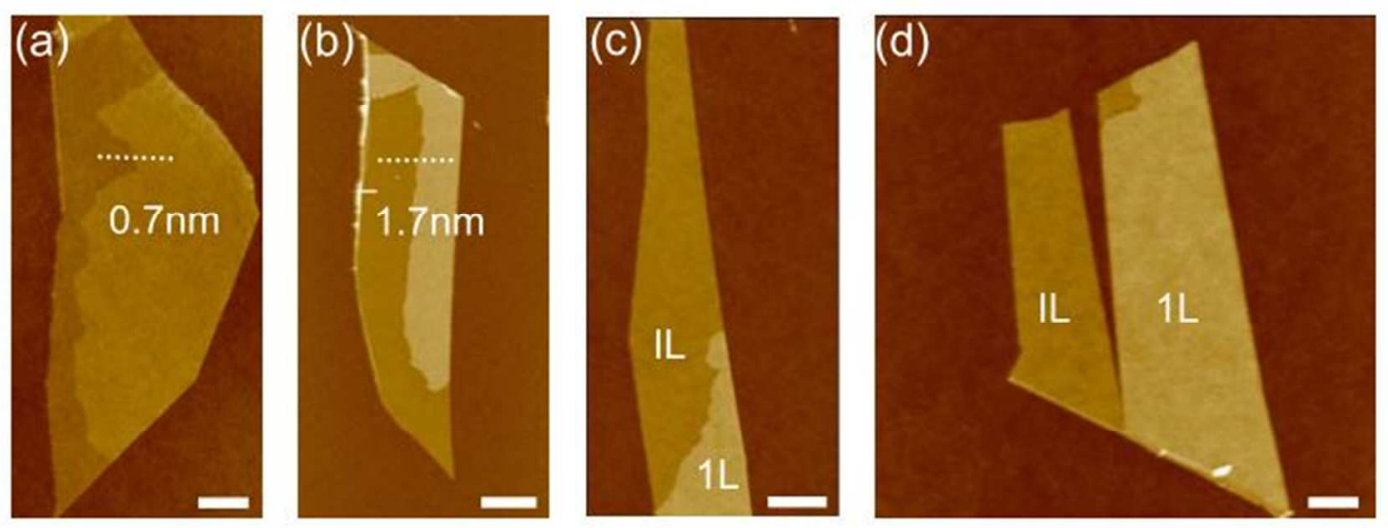

Figure S1. Layer-dependent thickness of $\mathrm{C}_{8}$-BTBT grown on graphene. AFM images of $\mathrm{C}_{8^{-}}$ BTBT grown on graphene, along with thickness measurement of (a)IL and (b)1L. (c)-(d) AFM images of two other incomplete $1 \mathrm{~L} \mathrm{C}_{8}$-BTBT on IL/graphene. Scale bars: $1 \mu \mathrm{m}(\mathrm{a}, \mathrm{d})$ and $2 \mu \mathrm{m}$ $(b, c)$. 


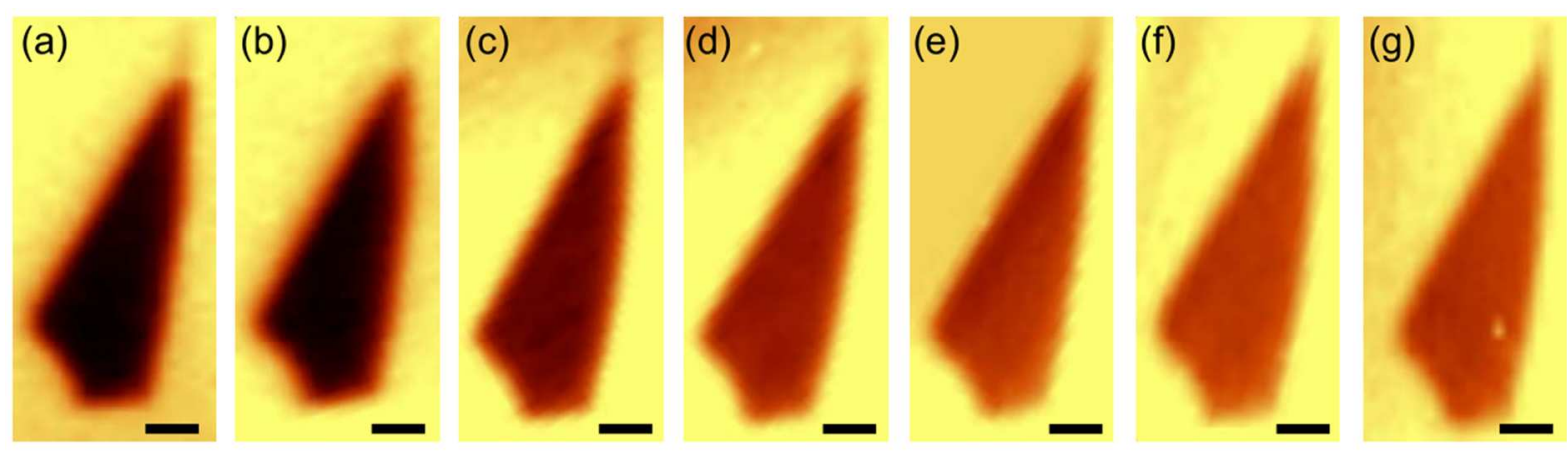

Figure S2. Polarization-dependent absorption images of the same sample in Fig. 2c. With respect to (a), the sample is rotated by (b) $15^{\circ}$, (c) $30^{\circ}$, (d) $45^{\circ}$, (e) $60^{\circ}$, (f) $75^{\circ}$ and (g) $90^{\circ}$, respectively. As we rotate the sample, the absorption is uniformly modulated, confirming the single-crystalline nature of the $\mathrm{C}_{8}$-BTBT film. All scale bars are $2 \mu \mathrm{m}$.
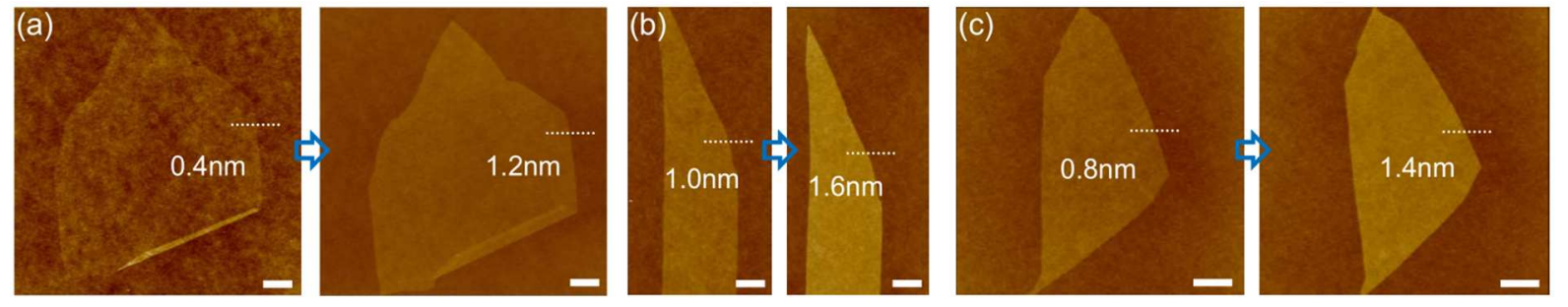

Figure S3. Three $\mathrm{C}_{8}$-BTBT samples placed in the same position $(10 \mathrm{~cm}$ away from the center) in Zone I with different growth times. AFM images of a graphene before (left) and after (right) growth for (a) 5 minutes, (b) 10 minutes and (c) 20 minutes, respectively. The increase of thickness by $0.6 \sim 0.8 \mathrm{~nm}$ indicates the complete coverage of monolayer $\mathrm{C}_{8}$ - $\mathrm{BTBT}$ on graphene in all three cases. Scale bars are $1 \mu \mathrm{m}$ for (a) and (b) and are $2 \mu \mathrm{m}$ for (c).
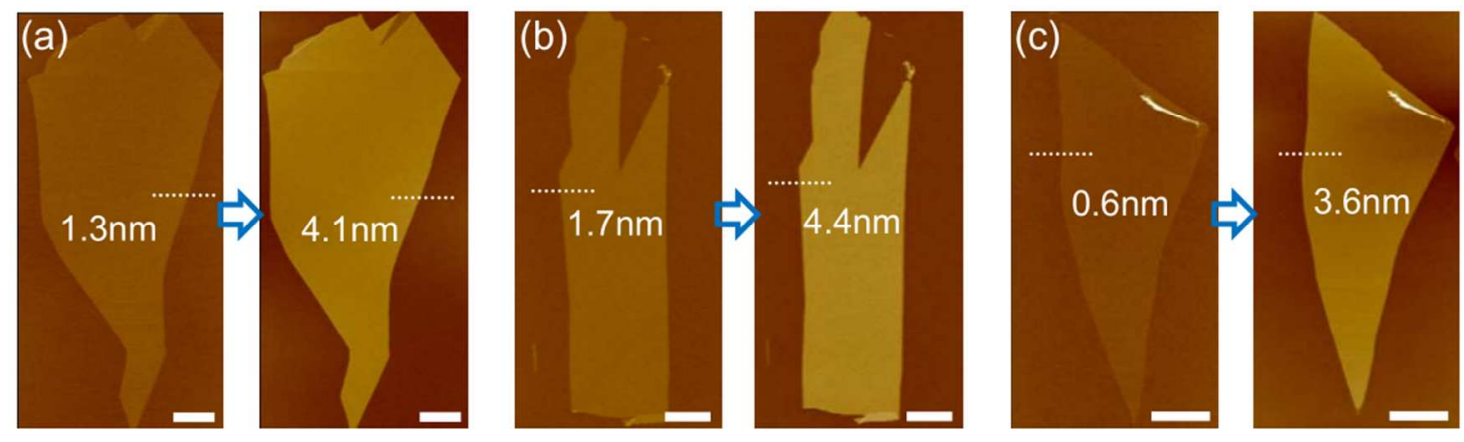
Figure S4. Three $\mathrm{C}_{8}-\mathrm{BTBT}$ samples placed in the same position $(12 \mathrm{~cm}$ away from the center) in Zone II with different growth times. AFM images of a graphene before (left) and after (right) growth for (a) 5 minutes, (b) 10 minutes and (c) 20 minutes, respectively. The increase of thickness by $2.7 \sim 2.8 \mathrm{~nm}$ indicates the complete coverage of bilayer $\mathrm{C}_{8}$ - $\mathrm{BTBT}$ on graphene in all three cases. Scale bars are $3 \mu \mathrm{m}$ for (a) and are $2 \mu \mathrm{m}$ for (b) and (c).
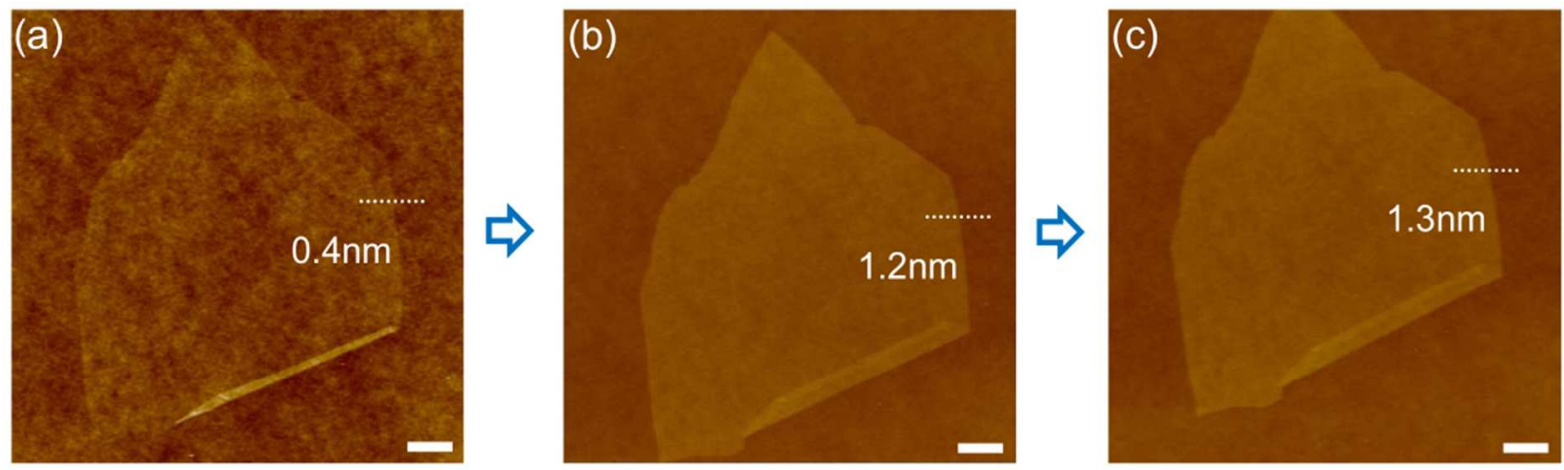

Figure S5. A monolayer $\mathbf{C}_{8}$-BTBT sample undergone repeated growths. (a) AFM image of (a) the graphene before growth, (b) after growth in Zone I (10 cm away from the center) for 5minutes and (c) after we repeat the growth for 5minutes. The repeated growth did not result in additional layers. Scale bars are $1 \mu \mathrm{m}$.
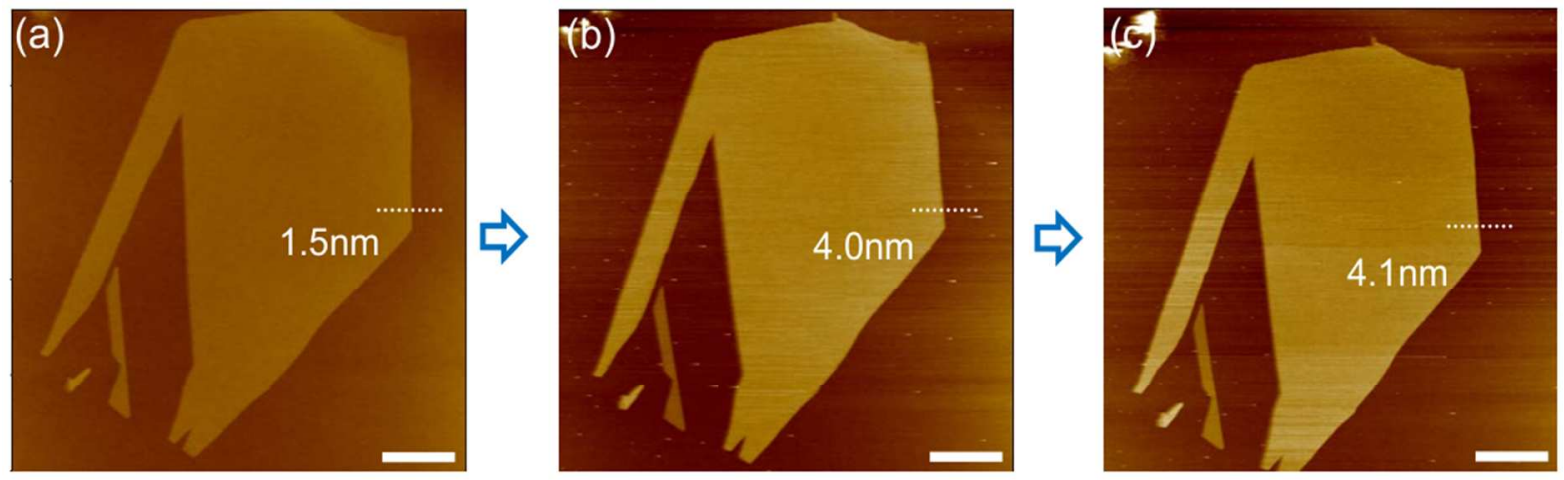

Figure S6. A bilayer $\mathbf{C}_{8}$-BTBT sample undergone repeated growths. (a) AFM image of (a) the graphene before growth, (b) after growth in Zone II (12cm away from the center) for 
5minutes and (c) after we repeat the growth for 5minutes. The repeated growth did not result in additional layers. Scale bars are $4 \mu \mathrm{m}$.
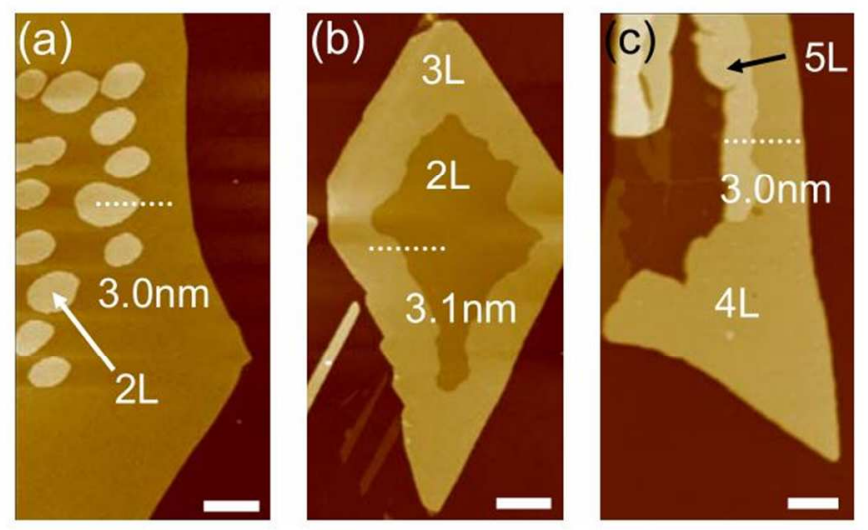

Figure S7. AFM images of three samples showing the constant height of $2 \mathrm{~L}, 3 \mathrm{~L}$ and $5 \mathrm{~L} \mathrm{C}_{8^{-}}$ BTBT. Scale bars are (a) $2 \mu \mathrm{m}$, (b) $1 \mu \mathrm{m}$ and (c) $1 \mu \mathrm{m}$.
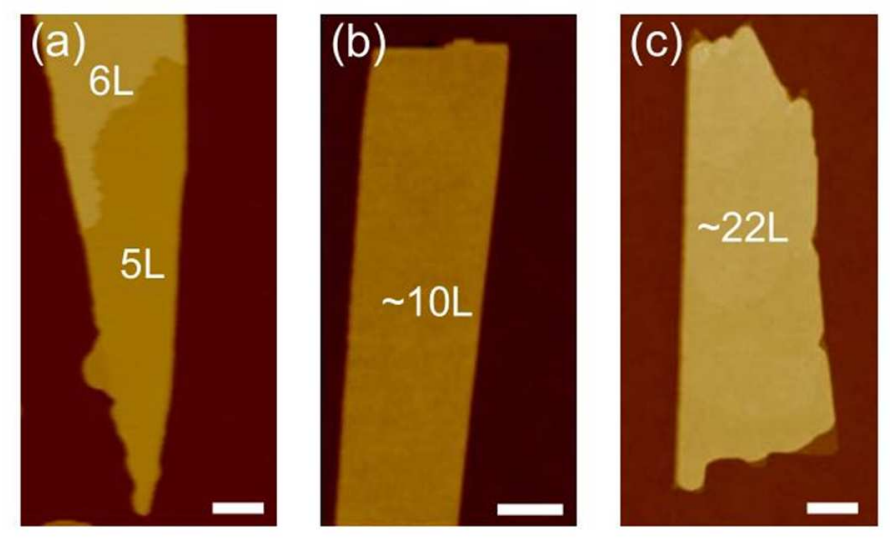

Figure S8. AFM images of uniform thicker films of $\mathrm{C}_{8}$-BTBT and PTCDA. (a) A multi-layer $\mathrm{C}_{8}$ - $\mathrm{BTBT}$ grown on $\mathrm{BN}$ substrate. The $\mathrm{BN}$ was placed $13 \mathrm{~cm}$ away from the center under $110^{\circ} \mathrm{C}$ for 20 minutes. (b) A multi-layer PTCDA grown on graphene substrate. The graphene was placed $15 \mathrm{~cm}$ away from the center under $270^{\circ} \mathrm{C}$ for 15 minutes. (c) A multi-layer PTCDA grown 
on graphene substrate. During growth, the graphene was placed $14 \mathrm{~cm}$ away from the center under $270^{\circ} \mathrm{C}$ for 20 minutes. Scale bars are $1 \mu \mathrm{m}$.

(a)

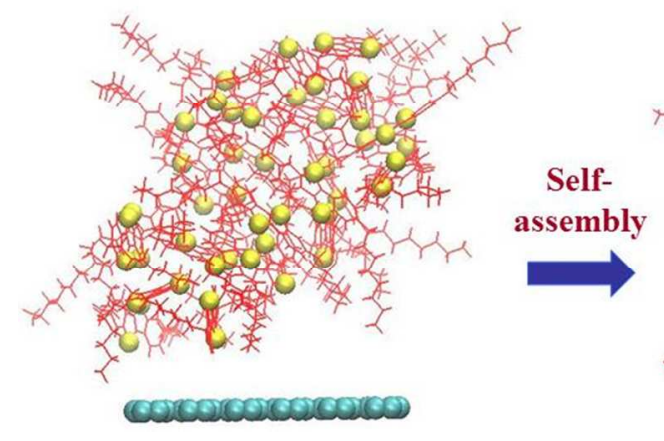

(b)

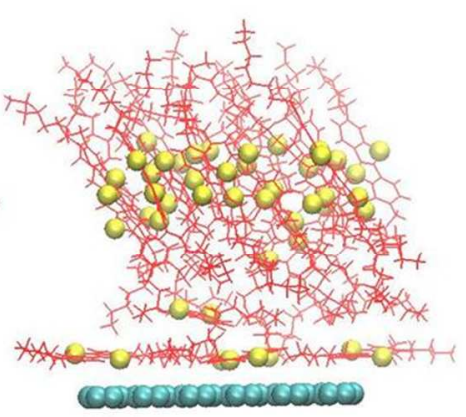

Figure S9. $\mathrm{C}_{8}$-BTBT self-assembly on graphene at $400 \mathrm{~K}$. (a) and (b) are initial and equilibrium configurations, respectively. The sulfur atoms of $\mathrm{C}_{8}$-BTBT are highlighted for clarity.
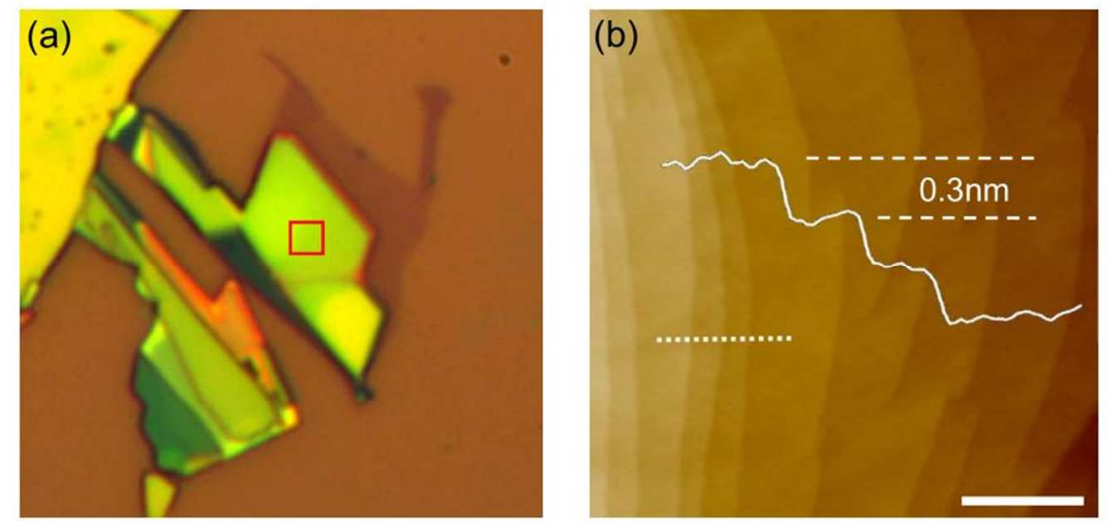

Figure S10. (a) Optical microscope images of a graphene sample after growth of bulk PTCDA ( $100 \mathrm{~nm}$ thick). (b) AFM image of the marked region in (a). PTCDA adopts layered structure with the thickness of each layer $\sim 0.3 \mathrm{~nm}$, indicating face-on packing. Scale bar is $500 \mathrm{~nm}$. 

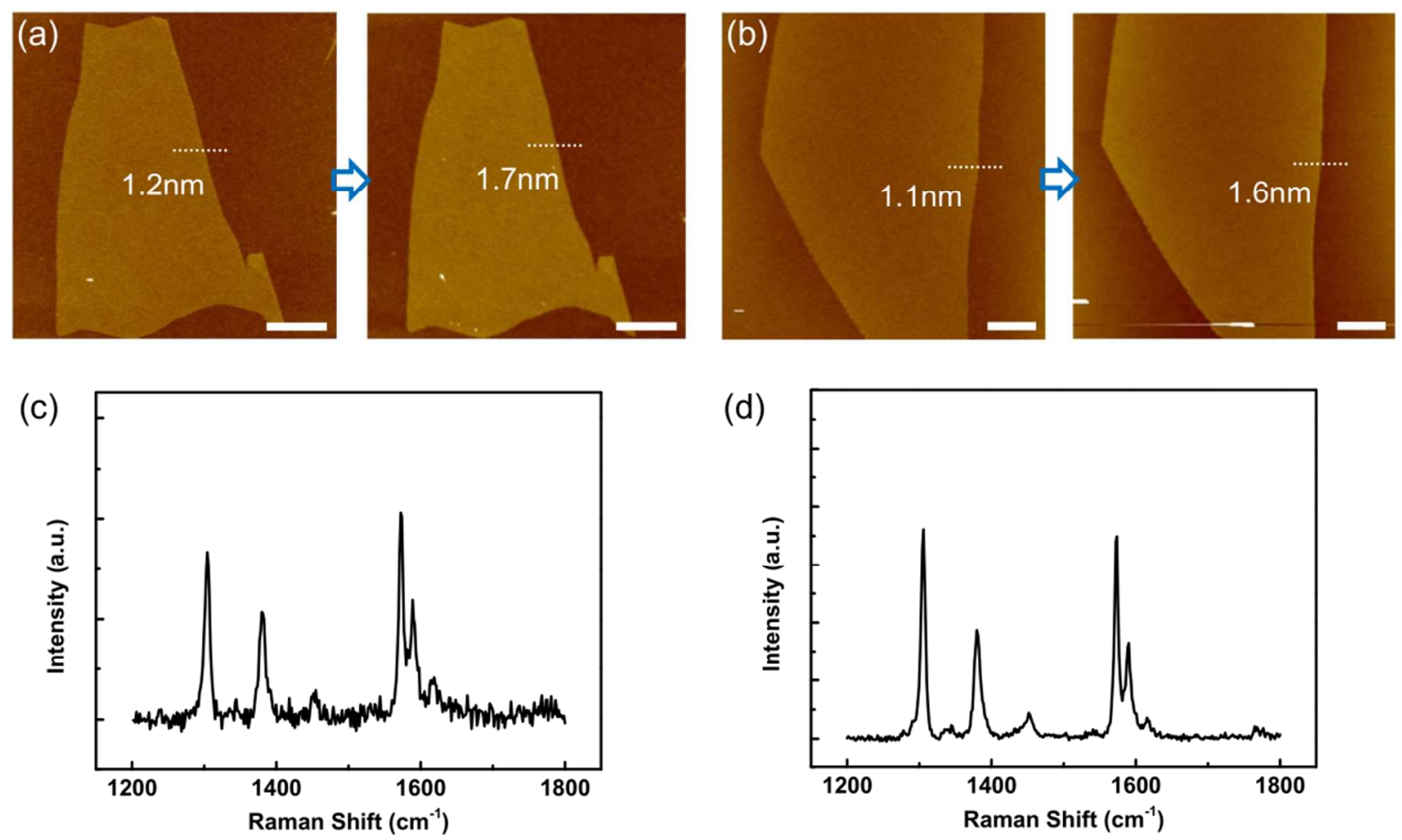

Figure S11. Two PTCDA samples placed in the same position ( $2 \mathrm{~cm}$ away from the center) with different growth times. The growth temperature is $280^{\circ} \mathrm{C}$ for both cases. (a) AFM images of a graphene before (left) and after (right) growth for 5 minutes. After growth, the thickness of the sample was increased by $\sim 0.5 \mathrm{~nm}$ as expected for monolayer PTCDA. Scale bars are $1.5 \mu \mathrm{m}$. (b) AFM images of a graphene before (left) and after (right) growth for 30 minutes. With respect to (a), longer growth time did not result in additional layers. Scale bars are $3 \mu \mathrm{m}$. (c) Raman spectrum of the sample in (a), showing clear Raman fingerprints of PTCDA. (d) Raman spectrum of the sample in (b). 

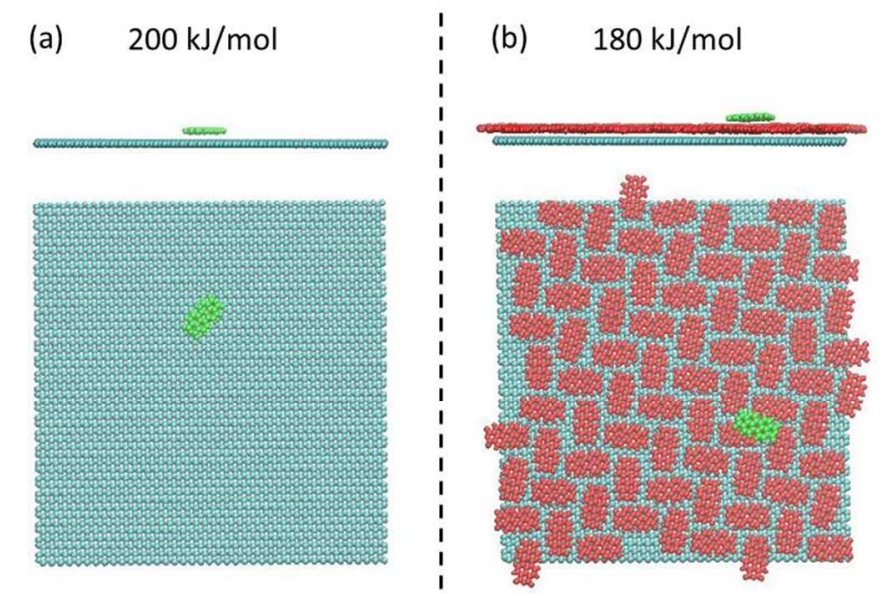

Figure S12. Binding energies and configurations of PTCDA on graphene (a) and IL/graphene (b).

\section{REFERENCES}

[1] Hess, B., Kutzner, C., van der Spoel, D., Lindahl, E. J. Chem. Theory Comput., 4, $435-447(2008)$

[2] Essmann, U., Perera, L., Berkowitz, M. L., Darden, T., Lee, H., Pedersen, L. G. J. Chem. Phys., 103, 8577-8593(1995).

[3] Wang, J. M., Wang, W., Kollman, P. A., Case, D. A. J. Mol. Graph. Model., 25, 247260(2006).

[4] Sousa da Silva, A. W., Vranken, W. F. BMC Research Notes, 5, 367(2012).

[5] Sorin, E. J., Pande, V. S. Biophys. J., 88, 2472-2493(2005).

[6] Guy, A. T., Piggot, T. J., Khalid, S. Biophys. J., 103, 1028-1036(2012).

[7] Zhang, Y. H. et al. Phys. Rev. Lett., 116, 016602(2016). 